\title{
Adaptation and Validation of the Tamil (Sri Lanka) Version of the Montreal Cognitive Assessment (MOCA)
}

\section{P.A.D. Coonghe ${ }^{1}$, P. Fonseka ${ }^{2}$, S. Sivayokan ${ }^{3}$, A. Kesavaraja ${ }^{4}$, R. Malhotra ${ }^{5}$, and T. Ostbye ${ }^{6}$}

\begin{abstract}
The study aimed to develop the Tamil (Sri Lanka) version of the Montreal Cognitive Assessment (MoCA) and investigate its reliability and validity as a brief screening tool for mild cognitive impairment (MCI). Tamil-speaking Sri Lankan older adults with normal cognition and MCI were recruited from a neurology clinic. Adaptation of the English MoCA to the Tamil (Sri Lanka) involved contextspecific content modification and translation. The content validity, reliability, sensitivity, and specificity of the tool were evaluated. Study participants were 184 older adults, comprising 85 with normal cognition and 99 neurologist-diagnosed MCI. The tool had high internal consistency (Cronbach's alpha=0.83). ROC curve analyses showed an area under the curve of 0.87 (95\% CI 0.83-0.91) for detecting MCI. The optimal cut-off score for detection of MCI was 23/24, yielded a sensitivity and specificity of $84.7 \%$ and $76.4 \%$, respectively. The Tamil (Sri Lankan) version of the MoCA maintains its core diagnostic properties rendering
\end{abstract}

1 Department of Community and Family Medicine, Faculty of Medicine, University of Jaffna, Sri Lanka, padcoonghe@univ.jfn.ac.lk

${ }^{2}$ No. 26 D 1/1, Rosmed Place, Colombo 7, Sri Lanka.

3 Psychiatry Unit, Teaching Hospital, Jaffna, Sri Lanka.

${ }^{4}$ Neurology Unit, Teaching Hospital, Jaffna, Sri Lanka.

${ }^{5}$ Duke-National University of Singapore (NUS) Medical School No.8, College Road, Singapore 169857.

${ }^{6}$ Duke Global Health Institute, No.310, Trent Drive, Durham NC USA, 27710.

Date Received: $22^{\text {nd }}$ October 2018

Date Accepted: $19^{\text {th }}$ December 2019 
it a valid and reliable tool for screening of MCI among Tamil speaking Sri Lankan older adults.

Key Words: Elderly, Jaffna, MCI, MoCA, Screening, Sri Lanka, Tamil

\section{Introduction}

Mild cognitive impairment (MCI) refers to an intermediate transitional cognitive phase between cognition of normal aging and mild dementia. Individuals with MCI carry a high risk of deterioration to Alzheimer's disease (AD) and other dementias relative to cognitively normal individuals. In 2005, the Montreal Cognitive Assessment (MoCA)(Nasreddine et al., 2005) was reported to be a better screening test for MCI than the Mini-Mental State Examination (MMSE)(Folstein, Folstein, \& McHugh, 1975) among English and Frenchspeaking persons. While the MoCA had a sensitivity and specificity of $90 \%$ and $87 \%$, respectively for detecting MCI, the MMSE, which is widely used by primary care physicians to screen for dementia, had a sensitivity of only $18 \%$ for MCI (Nasreddine et al., 2005). Consequently, the use of the MoCA for screening for MCI has become pervasive in many countries. At the same time translation and validation studies of the MoCA have confirmed its validity as a screening test for MCI in several countries, including South Korea, China and Sri Lanka (Lee et al., 2008, Yeung et al, 2014, Karunaratne et al, 2011).

In Sri Lanka, except for Sinhala language version of the MoCA (Karunaratne et al., 2011) the Tamil language version has not been validated.

Sri Lanka has a population of about 21million (Department of Census and Statistics, 2012). Sinhala and Tamil are the major languages spoken in the country with Tamil beins spoken by about 5 million individuals. Globally, Tamil is spoken by 75.8 million people, an official language in three countries (India, Sri Lanka, and Singapore) and is also common in Australia, Canada, Malaysia, Mauritius, and Burma. At the same time, many dialects of the spoken Tamil language, which vary from each other, are used in different geographical regions of the world (Simons \& Fennig, 2018). Such variations exist across regions in close geographical proximity; for instance, the vocabulary and grammar used by the 
speakers in Sri Lanka variy substantially from that is used by the Tamil speakers in India. For this reason, the Tamil version of the MoCA developed in India is not directly applicable to Sri Lanka. Further, details on the validity of the Tamil (India) version of the MoCA are not available. And, given the difference in literacy rates of the two countries (Sri Lanka: 92.6\% and India: 72.1\%) (UNESCO, 2013), and that educational level impacts the MoCA cut-off score for the detection of MCI, the Tamil (India) version of the MoCA (Nasreddine et al 2005 retrived from http://www.mocatest.org) may not be applicable to the Tamil-speaking population of older Sri Lankans. Thus, a culturally appropriate Sri Lankan Tamil version of the MoCA with acceptable psychometric properties is required to provide efficient and accurate screening of Tamil-speaking Sri Lankan older adults for MCI. The present study therefore aims to develop the Sri Lankan Tamil version of the MoCA and investigate its reliability and validity as a brief screening tool for MCI among Tamil-speaking Sri Lankan older adults.

\section{Methods}

\section{Participants}

This was a cross-sectional study of ambulatory, community-dwelling, Tamilspeaking Sri Lankan older adults recruited from the Neurology Clinic of the Teaching Hospital, Jaffna in Northern Sri Lanka. The approval of the research protocol from the Institutional Review Board (IRB), at the University of Jaffna was obtained. Written informed consent was obtained from all participants. All the MCI patients were able to give their consent without any difficulties. Based on the calculated sample size-a total of 184 older adults, comprising 85 with normal cognition and 99 neurologist-diagnosed MCI were recruited. For all participants, the inclusion criteria included age 60 years or older and the availability of an informant (i.e. a caregiver). The exclusion criteria included those with: cancer within 5 years, active infection, end-stage renal or other organ failure, non-ambulatory, depression by Diagnostic and Statistical Manual of Mental Disorders - fourth edition (DSM-IV, 2000) criteria, deafness, and other communication barriers. 


\section{Instrument}

The MoCA is a one-page instrument. It measures eight cognitive domains with 10 items, and includes tests on short-term memory recall, visuospatial abilities, multiple aspects of executive functions, phonemic verbal fluency, abstraction, attention, concentration and working memory, language function, and time and place orientation (http://www.mocatest.org). The MoCA can usually be completed in 10 minutes. In the original English and French MoCA version, one point is added for individuals with 12 or fewer years of education. The highest possible score is 30 points, and a higher score is indicative of better cognitive status.

We obtained permission to use the MoCA from its developer (Nasreddine et al., 2005). The original English version of the MoCA was first translated into (Sri Lanka) Tamil by three bilingual Tamil-speaking Sri Lankan medical professionals (Community Physician, Psychiatrist and Neurologist) separately, and the final version was approved with the consensus of all the experts who participated in the translation. Subsequently, this version was back-translated into English by a native Tamil speaker who was unfamiliar with the English version of the MoCA.

Finalizing the Sri Lanka Tamil version of MoCA, the following linguistic and cultural adaptations were made.

(1) Trail making test: The first five letters of the English alphabet were replaced with the first five letters of the Tamil Alphabet.

(2) Naming test: Pictures of a rhinoceros, and a camel were replaced with pictures of an elephant and a cow as the local community is more familiar with the latter animals than the former.

(3) Memory test: While two (face and red) of the five original words were retained, the rest (velvet, church, and daisy) were replaced. 'Velvet' was replaced with the Tamil word for 'Silk' as silk is a commonly used cloth material. 'Church' was replaced by the Tamil word for 'Temple' because most Tamil-speaking Sri Lankans are Hindus. And instead of 'Daisy', 'Jasmine', was selected. 
(4) Test of attention for letters: The English alphabet was replaced with Tamil letters that have a corresponding sound.

(5) Language repetition: The English version of MoCA has two sentences. The first sentence was translated to retain the same meaning and the same number of words. But the name 'John' was replaced with 'Kannan'. The second sentence was translated to retain the same number of words and also the same meaning without any corrections.

(6) Verbal fluency test: In the original tool, this test involves naming a maximum number of words beginning with the letter ' $F$ '. The corresponding Tamil ' $F$ ' is not used commonly. Therefore, it was replaced with the letter ' $\mathrm{P}$ '.

(7) Test for abstraction: This was included identification of similarity of three pairs . In the original tool, these pairs were 'Banana-Orange', 'Train- Bicycle', and 'Watch-Ruler'. During the process of adaptation, 'Banana- Orange' was retained without any change. But 'Train' was replaced by 'Bus' because the majority of the people in Northern Sri Lanka are unfamiliar with the train and bus as common public mode of travel Similarly, 'Watch' was replaced with 'Balance' because among older adults only a few use a watch and a weighing balance is a common measuring item that people are likely to have experienced.

The MoCA interviewer guide which has comprehensive instructions for administration of MoCA was also translated to Tamil. Necessary changes were made in MoCA-Tamil (Sri Lanka) adaptation. Then the translated guide was reviewed by a panel of experts who were involved in the translation into MoCATamil (Sri Lanka) of the original MoCA tool.

The adopted tool and guide were pre tested among ten patents from a medical clinic for chronic diseases at the Teaching Hospital, Jaffna and few corrections were made.

\section{Clinical diagnosis}

Older adults attending the Neurology Clinic of Teaching Hospital, Jaffna were subjected to full physical and neurological examination by a neurologist to classify 
them as having normal cognition or having MCI. Those deemed as having "normal" cognition were without any cognitive complaint or impairment on assessment and did not have dementia as per the DSM-IV criteria nor MCI. Those deemed as having MCI were classified so based on the criteria Winblad et al . (2004) i.e., the presence of memory complaint (corroborated by an informant), impaired memory function for age and education (delayed word recall score $<1$ SD below normal age- and education-matched mean for Sri Lankan older adults), intact activities of daily living and no dementia as per DSM-IV criteria. Every willing older adult ( $\mathrm{n}=184$; classified as either having normal cognition or having MCI) then underwent a detailed clinical and cognitive assessment, lasting for approximately 90 minutes. The results of the gold standard was blinded to the MOCA- Tamil (Sri Lanka) administration . Socio-demographic data, history on cognitive symptoms and coexisting diseases were collected and the MoCA-Tamil (Sri Lanka) was administered. Then, after 4 weeks, a total of 20 older adults (10 with normal cognition and 10 with MCI), selected from the 184 study participants were assessed for test-retest reliability of the MoCA by the same data collector. We also evaluated interrater reliability by inviting another subgroup of 20 participants (10 with normal cognition and 10 with $\mathrm{MCI}$ ), who were rated twice by two independent raters with the MoCA-Tamil (Sri Lanka).

\section{Statistical analysis}

The Original MoCA tool developers have suggested an additional item be added to persons with 12 years of education or less to minimize errors related to education. Therefore, in this study, an additional item was added to MoCA-Tamil (Sri Lanka) scores for those who had education for 12 years or less (if MoCATamil (Sri Lanka) score < 30). Demographic characteristics across those with normal cognition and MCI were compared using the chi-squared test (for categorical variables) or the student ' $t$ ' test (for continuous variables). Internal consistency reliability of the MoCA-Tamil (Sri Lanka) was assessed using Cronbach's alpha. Test-retest reliability of the MoCA-Tamil (Sri Lanka) was assessed using intra-class correlation coefficients (ICCs) for baseline and 4 weeks retest scores. ROC curve analysis was performed to determine sensitivity and 
specificity for detecting MCI. All tests considered two-sided hypotheses and a 5\% level of significance. SPSS for Windows (version 21.0 SPSS; SPSS, Chicago, IL, USA) was used for the analyses.

\section{Result}

\section{Study sample}

Study participants were 184 older adults, comprising 85 with "normal" cognition and 99 neurologist-diagnosed MCI. The mean age was 69.7(SD 4.8) years. There was no statistically significant difference in the mean age or sex between normal cognitive and MCI participants but, a significant difference was observed in years of education between the two categories of participants (Table1).

Table 2 shows the education-adjusted mean MoCA-Tamil (Sri Lanka) scores of "normal" cognitive and MCI participants. The mean MoCA-Tamil (Sri Lanka) score discriminated the two diagnostic categories of the study participants $(\mathrm{p}<0.001)$.

Table 1. Demographic characteristics of the Study Participants by cognitive status $(N=184)$

\begin{tabular}{llll}
\hline $\begin{array}{l}\text { Demographic } \\
\text { characteristics } \\
\text { Age, in years; Mean (SD) }\end{array}$ & Normal cognition & MCI & p-value \\
Years of education; & $9.2( \pm 2.7)$ & $70.2( \pm 5.0)$ & $\mathrm{p}^{\$}=0.12$ \\
Mean (SD) & $8.1( \pm 2.9)$ & $\mathrm{p}^{\$}=0.01$ \\
Male (\%) & $41(48.2)$ & $52(52.5)$ & $\mathrm{p}^{*}=0.56$ \\
Female (\%) & $44(51.8)$ & $47(47.5)$ & \\
\hline \$- $p$ value derived by student ' $t^{\prime}$ 'test $*$ p value derived by chi-square test
\end{tabular}


Table 2 Summary of Group Differences of MoCA-Tamil (Sri Lanka) scores

\begin{tabular}{llll}
\hline & \multicolumn{2}{c}{$\begin{array}{c}\text { MoCA-Tamil (Sri Lanka) Score } \\
\text { Normal Cognition }\end{array}$} & \multicolumn{1}{c}{ P value } \\
& \multicolumn{1}{c}{$\quad \begin{array}{c}\text { MCI (N=99) } \\
\text { (N=85) }\end{array}$} & \\
Mean score & 26.4 & 21.2 & $\mathrm{p}<0.01$ \\
$(95 \%$ CI $)$ & $(26.0-26.8)$ & $(20.7-21.7)$ & \\
Median & 26.0 & 21.0 & \\
Inter Quartile & $25-28$ & $19-23$ & \\
Range & & & \\
Skewness & 0.30 & -0.09 & \\
\hline
\end{tabular}

\section{Reliability of MoCA-Tamil (Sri Lanka)}

Test - retest reliability data were collected from a subsample of 20 participants (Normal cognition and MCI) tested, on average, $28 \pm 1.5$ days apart. The mean change in MoCA-Tamil (Sri Lanka) scores from the first to second evaluation was $0.8 \pm 1.8$ points, and the correlation between the two evaluations was high (correlation coefficient $=0.93, \mathrm{p}<0.001$ ) and suggesting good stability over time .

\section{Internal consistency of MoCA-Tamil (Sri Lanka)}

Internal consistency of the scale was assessed using Cronbach's alpha. An alpha value of 0.7-0.9 was considered as evidence to support good internal consistency of the instrument (Streiner, 1993). The internal consistency of the MoCA-Tamil (Sri Lanka) was moderate to high, yielding a Cronbach's alpha of 0.831. This indicated good internal consistency. 
Table 3. Item Total Statistics Analysis of MoCA Tamil (Sri Lanka)

\begin{tabular}{llll}
\hline & $\begin{array}{l}\text { Scale mean if } \\
\text { item deleted }\end{array}$ & $\begin{array}{l}\text { Correlated } \\
\text { item- total } \\
\text { correlation }\end{array}$ & $\begin{array}{l}\text { Cronbach's } \\
\text { alpha if item } \\
\text { removed }\end{array}$ \\
\hline Trail making & 19.1304 & 0.525 & 0.782 \\
Copy cube & 19.2536 & 0.404 & 0.790 \\
Clock drawing & 17.5145 & 0.688 & 0.751 \\
Naming & 16.5870 & 0.214 & 0.798 \\
Digit span & 18.2681 & 0.560 & 0.773 \\
Sustained attention & 18.8841 & 0.659 & 0.774 \\
Serial 7 calculation & 17.3333 & 0.551 & 0.770 \\
Sentence repetition & 18.3949 & 0.442 & 0.784 \\
Phonemic fluency & 19.3370 & 0.325 & 0.794 \\
Abstraction & 17.8913 & 0.575 & 0.774 \\
Delayed recall & 17.3623 & 0.378 & 0.819 \\
Orientation & 14.2246 & 0.563 & 0.769 \\
\hline Scale statistics & 19.4710 & & 0.831 \\
\hline
\end{tabular}

\section{Inter-observer reliability}

The inter-observer reliability was assessed through the application of the test on the first ten patients in the study by two independent blinded evaluators. A correlation of coefficient and concordance of 0.91 was obtained with a $95 \%$ CI of $(0.77,0.99)$. This correlation and concordance coefficient are considered almost perfect.

According to Table 3. which shows item test statistics, the correlated item- total correlations are basically the correlation between the particular item and a composite score of all the other remaining items. In addition, if the corrected itemtotal correlation is $>0.30$, it indicates homogeneity between each item and total inventory score. Values $<0.30$ indicate that a particular item correlates poorly with the overall scale (Abdollahimohammad \& Ja'afar, 2014). In this tool, all the items 
except Naming have higher than expected corrected item- total correlation. 'Cronbach's Alpha, if item removed; is a measure of examining the relationship between the individual item and the total scale. This is the value of Cronbach's alpha for the remaining items if the given item is not included in the scale. So, in this scale, all the subscales' 'Cronbach's Alpha if item removed' are lower than overall scale's Cronbach's alpha (0.831).

Table 4. Sensitivity, specificity, positive predictive value (PPV) and negative predictive value (NPV) of the MoCA-Tamil (Sri Lanka) for detection of normal cognitive and MCI patients

\begin{tabular}{lllll}
\hline Cutoff & Sensitivity & Specificity & PPV (\%) & NPV (\%) \\
\hline$\geq 21$ & 52.4 & 100.0 & 100.0 & 48.32 \\
$\geq 22$ & 58.6 & 100.0 & 100.0 & 51.80 \\
$\geq 23$ & 68.1 & 97.6 & 98.45 & 57.60 \\
$\geq \mathbf{2 4}$ & $\mathbf{8 4 . 7}$ & $\mathbf{7 6 . 4}$ & $\mathbf{8 8 . 9 0}$ & $\mathbf{6 8 . 4 0}$ \\
$\geq 25$ & 87.7 & 64.7 & 84.80 & 70.00 \\
$\geq 26$ & 90.6 & 44.7 & 78.60 & 67.9 \\
$\geq 27$ & 94.8 & 28.2 & 76.70 & 70.79 \\
$\geq 28$ & 97.4 & 16.5 & 72.38 & 73.85 \\
$\geq 29$ & 100.0 & 7.1 & 58.30 & 100.00 \\
$\geq 30$ & 100.0 & 0.0 & 69.2 & Not \\
& & & & applicable
\end{tabular}

$\overline{\text { Bold values indicate cutoff point used in the study with values of sensitivity and }}$ specificity.

\section{Predictive validation}

With the use of a cut-off of 26 points (suggested in the literature as the ideal MoCA cut-off score to detect MCI), the MoCA-Tamil (Sri Lanka) detected 90.6\% of MCI cases, but specificity was reduced to $44.7 \%$ (Table 4 ). With the use of the cut-off of 24 points, the MoCA-Tamil (Sri Lanka) exhibited high sensitivity (84.7\%) and 
specificity $(76.4 \%)$. Therefore, 24 points seemed to provide the best balance between sensitivity and specificity (any score of $\leq 23$ was considered to be the abnormal result).

The area under the ROC curve (AUC) of the MoCA-Tamil (Sri Lanka) for the identification of MCI was 0.87 (95\% CI 0.83-0.91) (Fig.1)

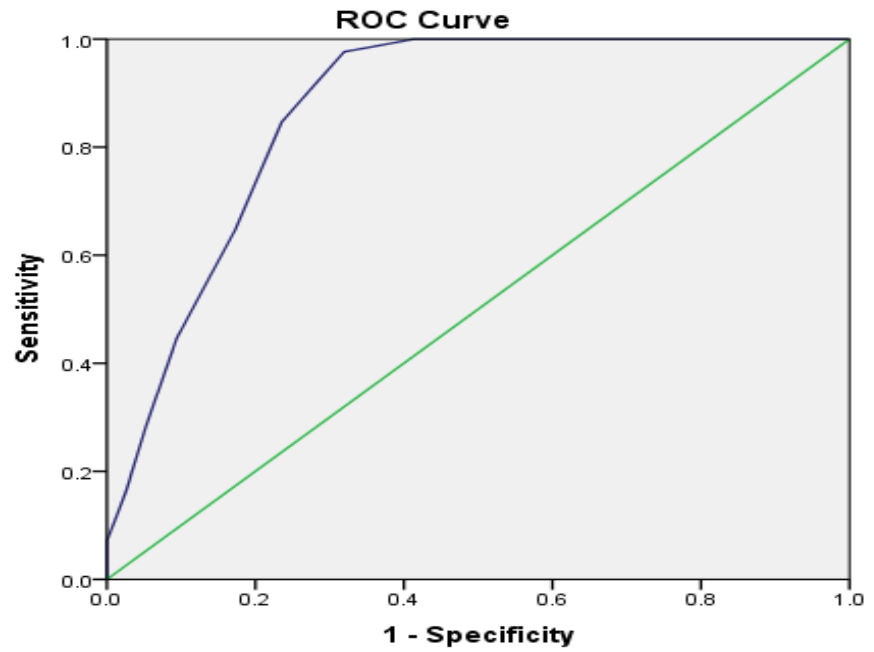

Figure 1 Receiver Operating Characteristic (ROC) curve analysis of MoCA-Tamil (SriLanka) for the detection of normal cognitive and $\mathrm{MCl}$ patients

\section{Discussion}

The objective of this study was to develop the Tamil (Sri Lanka) version of the MoCA and investigate its reliability and validity as a brief screening tool for MCI among Tamil-speaking Sri Lankan older adults. In Sri Lanka the majority of the population is rapidly ageing. The validation of the MoCA in Tamil (Sri Lanka) will be very useful for the clinicians and especially for the primary care physicians in detecting MCI in order to enhance the provision of appropriate care in time. The results of this study seem to be in line with previous validation studies using the MoCA. First, the MoCA Tamil (Sri Lanka) mean scores for the diagnostic groups were similar to the ones presented in the original study (Nasreddine et al., 2005).Second, the MoCA-Tamil (Sri Lanka) demonstrated adequate test-retest 
reliability and high internal consistency and these were similar to the studies of MoCA validation by Nasreddine et al., 2005; Gil, Vega, Ruiz de Sanchez, \& Burgos, 2013; Karunaratne et al., 2011; Yu et al., 2012.

As shown in Table 3. 'Cronbach's Alpha if item removed' is a measure of examining the relationship between individual item and the total scale. This is the value of Cronbach's alpha for the remaining items if the given item is not included in the scale. So, in this scale all the subscales' 'Cronbach's Alpha if item removed' are lower than overall scale's Cronbach's alpha (0.831). It means, all the items are needed for the scale in terms of reliability. Karunaratne et al., (2011) found that Cronbach's alpha increased when the item naming was removed and they concluded that the contribution of the item 'Naming' to the scale was poor.

Sensitivity and specificity of a tool were determined based on the cutoff point decided with the help of ROC curve. A score which yields the best balance between sensitivity and specificity for the MCI participants was decided as the cutoff score. As described in Table 4 the cutoff score of $\geq 24$ discriminate NC from MCI participants with the sensitivity of $84.7 \%$ and specificity of $76.4 \%$. This cutoff score provides the positive predictive value (PPV) as $88.9 \%$ and negative predictive value (NPV) as $68.4 \%$. When increasing the cut-off value to 26 as recommended by the authors of the original MoCA tool the sensitivity decreased to $68.1 \%$ but specificity increased to $97.6 \%$. Because of this reason, it was decided that a score of $\geq 24$ was the cut-off value to detect NC from the MCI participants.

The Spanish version of the MoCA validation used scores of $\geq 23$ with the optimal sensitivity (89\%) and specificity (79.8\%) (Gil et al., 2013). In the Beijing version of the Chinese MoCA validation, the cut-off score for the optimal sensitivity and specificity to detect MCI appeared to be $\geq / 22$ for the MoCA-BJ, at which the sensitivity and specificity were $68.7 \%$ and $63.9 \%$ respectively (Yu et al., 2012).

Some limitations of this study should be mentioned. This tool cannot be applied to illiterate elders, and therefore they were excluded from the study. Another limitation is the fact that the MCI group was heterogeneous, as different MCI 
subtypes were included in the sample. In addition, this was not a community study, as the sample was hospital based.

\section{Conclusion}

The Validated Tamil (Sri Lanka) MoCA is a reliable and an acceptable tool to assess MCI in the community setting with a cut-off value of $\geq 24$. It had $84.7 \%$ sensitivity and $76.4 \%$ Specificity. The reliability and internal consistency of the tool were higher than the expected level.

\section{References}

Abdollahimohammad, A., \& Ja'afar, R. (2014). Learning Style Scales: a valid and reliable questionnaire. Journal of Educational Evaluation for Health Professions, 11, 22. https://doi.org/10.3352/jeehp.2014.11.22

Department of Census and Statistics, S. L. (2012). Census of Population and Housing of Sri Lanka , 2012 Population by divisional secretariat division , sex.

Diagnostic and Statistical Manual of Mental Disorders, Fourth Edition, Text Revision. Washington, D.C., American Psychiatric Association, 2000. (2000). Text, 2000.

Folstein, M. F., Folstein, S. E., \& McHugh, P. R. (1975). “Mini-mental state”. A practical method for grading the cognitive state of patients for the clinician. Journal of Psychiatric Research, 12 (3), 189-198. Retrieved from http://www.ncbi.nlm.nih.gov/pubmed/1202204

Gil, L., Vega, J. G., Ruiz de Sanchez, C., \& Burgos, F. P. (2013). Validation of the Montreal Cognitive Assessment - Spanish Version test (MoCA-S) as a screening tool for mild cognitive impairment and mild dementia in Bogotá, Colombia. Alzheimer's \& Dementia, 9 (4), P452- P453. https:// doi.org/ 10.1016/ j.jalz. 2013.05.904

Karunaratne, S., Hanwella, R., \& De Silva, V. (2011). Validation of the Sinhala version of the Montreal Cognitive Assessment in screening for dementia. Ceylon Medical Journal, 56(4), 2-8. https://doi.org/10.4038/cmj.v56i4.3892 
Lee, J.-Y., Dong Woo Lee, Cho, S.-J., Na, D. L., Hong Jin Jeon, Kim, S.-K., ... Maeng Je Cho. (2008). Brief screening for Mild Cognitive Impairment in Elderly Outpatient Clinic: Validation of the Korean version of the Montreal Cognitive Assessment. Journal of Geriatric Psychiatry and Neurology, 21(2), 104-110. https://doi.org/10.1177/0891988708316855

Nasreddine, Z. S., Phillips, N. a., Bédirian, V., Charbonneau, S., Whitehead, V., Collin, I., ... Chertkow, H. (2005). The Montreal Cognitive Assessment, MoCA: A brief screening tool for mild cognitive impairment. Journal of the American Geriatrics Society, 53(4), 695-699. https://doi.org/10.1111/j.1532-5415. 2005. 53221.x

Simons, G. F., \& Fennig, C. D. (2018). Ethnologue: Languages of the world. Retrieved February 2, 2019, from https://www.ethnologue.com/language/tam

Streiner, D. L. (1993). A checklist for evaluating the usefulness of rating scales. Canadian Journal of Psychiatry. Revue Canadienne de Psychiatrie, 38(2), 140148. Retrieved from http://www.ncbi.nlm.nih.gov/pubmed/8467441

UNESCO, N. D. (2013). UNESCO country programming document for Sri Lanka, 2013-2017; 2013. Retrieved from http://unesdoc.unesco.org /images/ 0022/002242/224243E.pdf

Winblad, B., Palmer, K., Kivipelto, M., Jelic, V., \& Fratiglioni, L. (2004). Introduction: Mild cognitive impairment: Beyond controversies, towards a consensus. Journal of Internal Medicine, 256(3), 181-182. https://doi.org/10.1111/j.1365-2796.2004.01382.x

Yeung, P. Y., Wong, L. L., Chan, C. C., Leung, J. L. M., \& Yung, C. Y. (2014). A validation study of the Hong Kong version of Montreal Cognitive Assessment (HK-MoCA) in Chinese older adults in Hong Kong. Hong Kong Medical Journal, 20(6), 504-510. https://doi.org/10.12809/hkmj144219 